\section{DGD monitoring issues in high-speed polarisation multiplexed coherent QPSK systems}

\author{
E. Matarazzo, A. Peracchi, R. Corsini, G. Meloni, L. Poti, \\ R. Magri and E. Ciaramella
}

\begin{abstract}
Optical performance monitoring based on digital signal processing (DSP) of a polarisation diverse coherent receiver can allow the estimation of the differential group delay (DGD) of the link fibre. In this reported work, it was found that the DGD estimation can be affected by substantial errors, since the DSP can introduce or leave uncompensated any symbol-time delay between orthogonal polarisations and cannot discriminate the in-line DGD from any other link delay. These issues are investigated and a method is proposed to partially overcome the problems.
\end{abstract}

Introduction: The recent progress in high-speed DSP for electronic signal processing has allowed for coherent systems based on digital equalisation at the receiver [1]. The adaptive two-dimensional (2D) fractionally spaced feed-forward equaliser (FFE), if properly dimensioned, converges to the $2 \mathrm{D}$ matched filter $[1,2]$, achieving perfect compensation of the channel and thus allowing a simple classical symbol-bysymbol detection [3]. The baseband representation of the optical field (amplitude and phase) in the electrical domain together with the following digitisation leads to a greater effectiveness of all post-detection processing techniques. In fact, the digital equaliser structure not only is able to compensate for all deterministic linear channel impairments, but can also enable a comprehensive optical performance monitoring (OPM) [4, 5], which provides information about the fibre linear parameters in a simple, cost- and power-effective way. Among the various fibre parameters, the estimation of the differential group delay (DGD) was found to be affected by a non-negligible offset and fluctuations $[4,6$, 7]. Some of those fluctuations were explained because of a combination with polarisation dependent loss (PDL) [4]. In this Letter, we demonstrate that DGD estimation errors also arise from other sources, and we found these even without PDL. We first use Monte Carlo simulations to check the DGD estimation technique in a $100 \mathrm{Gbit} / \mathrm{s}$ polarisation multiplexed quadrature phase shift keying (PM-QPSK) system. In this case, we observe incorrect DGD estimations, which we ascribe to the fact that the DSP can introduce or leave uncompensated any symboltime delay between orthogonal polarisations. We solve this issue by exploiting the information from the pattern of the two orthogonally polarised signals. We then test experimentally the technique in a $112 \mathrm{Gbit} / \mathrm{s}$ coherent test-bed; here we observed that the estimation is also critically affected by any other spurious delay present in the transmitter and/or in the receiver.

Simulation and experimental results: To investigate the DGD monitoring, we first perform extensive Monte Carlo simulations. We used the system model presented in [3] and, after the convergence was obtained, we extracted the values of the FFE taps. These were processed to get the DGD estimation. We found two different problems. First, the final equaliser configuration is not uniquely defined: clearly, the convergence properties of the stochastic-gradient algorithm for the adjustment of the tap-weight values are such that the FFE equaliser can introduce an arbitrary delay between the two PolMux signals equal to an integer multiple of the symbol time $\mathrm{T}_{\mathrm{s}}(=40 \mathrm{ps}$ in a $100 \mathrm{Gbit} / \mathrm{s}$ PM-QPSK system). In that case, the estimated DGD value from the FFE taps is thus misleading, as it is given by the sum of the DGD of the link and of the above delay due to the equaliser. Furthermore, if the link DGD is equal to an integer multiple of $\mathrm{T}_{\mathrm{s}}$, the FFE may not invert the channel realigning the two orthogonal polarisations, then the resulting sequences have a synchronisation delay of one or more symbol times and the estimated DGD value is (erroneously) close to $0 \mathrm{ps}$. Both these effects are clearly represented by dotted lines in Fig. 1: here we show the DGD estimated values for different fibre DGD values and FFE taps number. As can be seen, in many cases the estimated DGD is not correct. Moreover, when the fibre DGD is 40 or $80 \mathrm{ps}\left(\mathrm{T}_{\mathrm{s}}\right.$ or $\left.2 \mathrm{~T}_{\mathrm{s}}\right)$, both types of FFE erroneously estimate a DGD value close to $0 \mathrm{ps}$. We outline that in these cases, the FFE is perfectly working: indeed the system is showing good BER performance.

From the above discussion, we see that we can correct the estimated DGD value if the monitoring unit knows the delay induced by the equaliser between the two polarisations. To do this, in the simulations we exploit the knowledge of the transmitted symbols (in a practical case one can exploit the known features of the OTN frame structure) If we apply this delay correction to the estimated DGD values, the corrected values agree well with the fibre values: the estimation error is lower than $10 \%$ using five taps ( $<1 \%$ using 21 taps) as shown by the solid curves in Fig. 1.

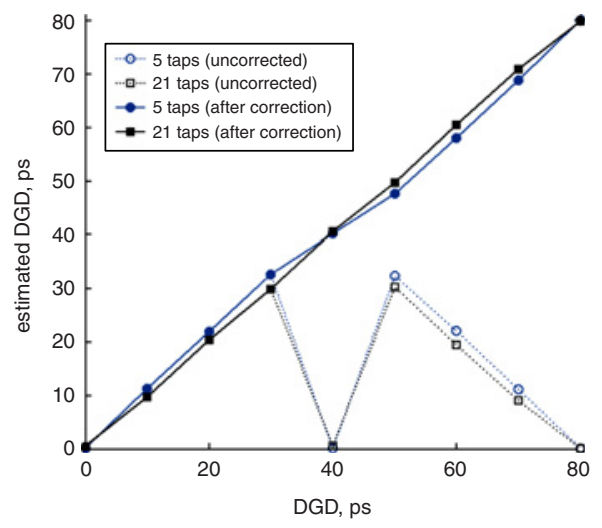

Fig. 1 Numerical simulation results

We report estimated DGD for different number of taps against given fibre value before (dotted lines) and after delay correction (solid lines) In all cases, $E_{\mathrm{b}} / N_{0}=10 \mathrm{~dB}$. Two FFEs are considered with five and 21 taps, respectively

To experimentally validate these results, we performed the DGD estimation in a $112 \mathrm{Gbit} / \mathrm{s}$ PM-QPSK optical coherent system. The experimental setup is shown in Fig. 2. Four pseudorandom bit sequences (PRBS) at $7 \mathrm{Gbit} / \mathrm{s}$ are combined through a multiplexer (RF MUX). The obtained $28 \mathrm{Gbit} / \mathrm{s}$ electrical data stream is power-divided and one of the two branches is delayed by 48 symbol times. The two RF signals are then sent to the $\mathrm{I}$ and $\mathrm{Q}$ input of the $\mathrm{I} / \mathrm{Q} \mathrm{LiNbO}_{3}$ modulator. An external cavity tunable laser is used as the optical source at a wavelength $\lambda=1548 \mathrm{~nm}$. The $56 \mathrm{Gbit} / \mathrm{s}$ quaternary phase shift keying QPSK signal is then sent to a polarisation multiplexer (PolMux) emulator where it is split into two components. One of them is delayed by 112 symbol times, rotated in polarisation by $90^{\circ}$ and then coupled together to the other, thus obtaining a $112 \mathrm{Gbit} / \mathrm{s}$ PM-QPSK signal. A DGD emulator (JDSU PE4) is used to introduce a variable DGD after the transmitter. At the receiver side, the signal and the local oscillator are sent to a polarisation diversity $90^{\circ}$ hybrid coupler, and detected by four balanced receivers. The four electrical signals are sampled by a $50 \mathrm{GSample} / \mathrm{s}$ real-time oscilloscope, and then processed offline on a common PC. Fig. 3 shows the estimated DGD against the DGD introduced by the emulator. The experimental results confirm the simulations: the estimated DGD is sometimes misleading. The experimental data are reported in Fig. 3. Here the stars represent the data affected by synchronisation delay (integer multiple of the symbol time $\mathrm{T}_{\mathrm{s}}=35.7 \mathrm{ps}$ ) and circles represent the data obtained in the case of correct synchronisation. Analysing in detail the data not affected by synchronisation errors (circles in Fig. 3), we observe that they still suffer from non-negligible error with a maximum value of $6 \mathrm{ps}$. To investigate the source of such an uncertainty, we performed 100 measurements of the system in back-to-back configuration, i.e. without any added DGD. The obtained DGD data distribution has a mean value of $6.6 \mathrm{ps}$ with very low standard deviation $(1.4 \mathrm{ps})$. This means that the system has some other delays due to the non-ideality of the transmitter, receiver, and all the other components in the experimental setup. As an example, one source of this type of delay was found to be the PolMux device (having around 5 ps more delay than the exact multiple number of symbols between the two branches). Again, these effects do not impair the system performance. Actually, when the equaliser inverts the channel, it compensates for all these delays and the fibre DGD randomly combines. Consequently, the DGD value estimated by the OPM includes not only the contribution of the line. Whilst the previous synchronisation delays can be easily compensated for, these spurious delays cannot be separated from the DGD of the link, thus making the exact DGD estimation almost impossible. 


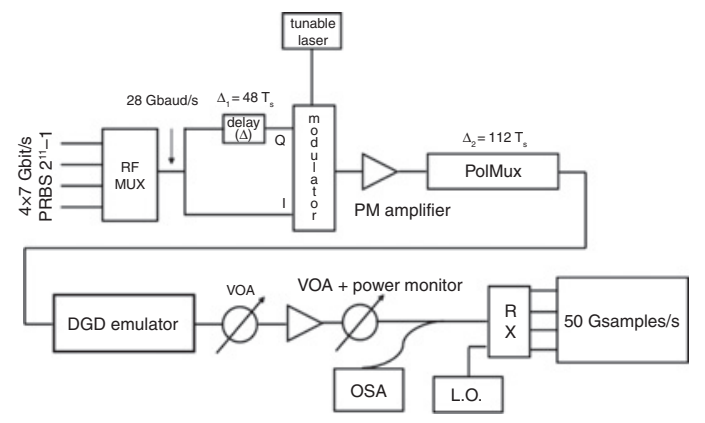

Fig. 2 Experimental setup

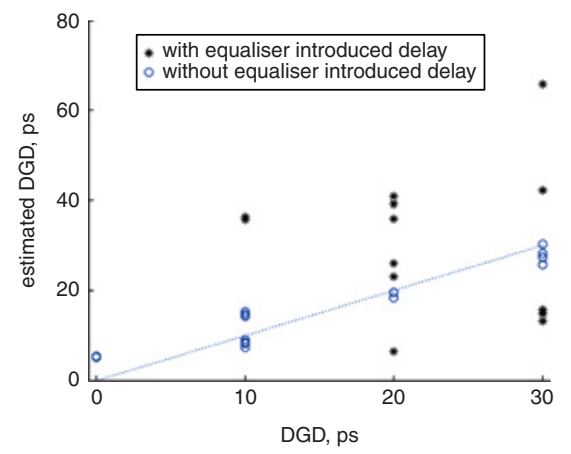

Fig. 3 Experimentally estimated DGD with $\left(^{*}\right)$ and without delay correction (o) against given $D G D$ value (OSNR $=40 \mathrm{~dB}$ and 11 taps)

Conclusions: We have investigated the practical issues that can limit the DGD estimation in coherent systems. The first source of errors is that, in some cases, the FFE inverts the channel unless integer multiples of $T_{s}$ : then the estimated value is misleading. We solve this problem by checking the frame synchronisation of the two PolMux signals. However, when moving from the simulation to the lab tests, we found another source of errors, i.e. the DGD estimation is sensitive to any other delay that can be present in the real setup, e.g. generated by the nonideal transmitter, receiver and components. These delays randomly combine with the link DGD and can give a misleading estimation. In both cases, these effects have an impact on the DGD estimation, but do not affect the system performance.

(C) The Institution of Engineering and Technology 2012

19 January 2012

doi: $10.1049 / \mathrm{el} .2012 .0214$

One or more of the Figures in this Letter are available in colour online.

E. Matarazzo, A. Peracchi, R. Corsini, G. Meloni and E. Ciaramella (Scuola Superiore Sant'Anna di Studi Universitari $e$ di Perfezionamento, Via G. Moruzzi 1, Pisa 56124, Italy)

E-mail: e.matarazzo@sssup.it

L. Potì (CNIT Research Unit, Via G. Moruzzi 1, Pisa 56124, Italy)

R. Magri (Ericsson, Via G. Moruzzi 1, Pisa 56124, Italy)

\section{References}

1 Winters, J.H.: 'Equalization in coherent lightwave systems using a fractionally spaced equalizer', J. Lightwave. Technol., 1990, 8, (10), pp. 1487-1491

2 Taylor, M.G.: 'Coherent detection method using DSP for demodulation of signal and subsequent equalization of propagation impairments', IEEE Photonics Technol. Lett., 2004, 16, (2), pp. 674-676

3 Colavolpe, G., Foggi, T., Forestieri, E., and Prati, G.: 'Robust multilevel coherent optical systems with linear processing at the receiver', J. Lightwave. Technol., 2009, 27, (13), pp. 2357-2369

4 Hauske, F.N., Kuschnerov, M., Spinnler, B., and Lankl, B.: 'Optical performance monitoring in digital coherent receivers', J. Lightwave. Technol., 2009, 27, (16), pp. 3623-3631

5 Faruk, Md.S., Mori, Y., Zhang, C., Igarashi, K., and Kikuchi, K.: "Multi-impairment monitoring from adaptive finite-impulse-response filters in a digital coherent receiver', Opt. Express, 2010, 18, (26), pp. $26929-26936$

6 Woodward, S.L., Nelson, L.E., Magill, P.D., Foo, S., Moyer, M., and O'Sullivan, M.: 'A shared PMD and chromatic dispersion monitor based on a coherent receiver', IEEE Photonics Technol. Lett., 2010, 22, (10), pp. 706-708

7 Geyer, J.C., Fludger, C.R.S., Duthel, T., Schulien, C., and Schamauss, B.: 'Optical performance monitoring using a $43 \mathrm{~Gb} / \mathrm{s}$ realtime coherent receiver'. Summer Topical Meeting, Piscataway, NJ, USA, 2009 (LEOSST '09. IEEE/LEOS), pp. 93-94 